\title{
Antibiogram and Antimicrobial Susceptibility Pattern of Bacterial Isolates from a Tertiary Care Hospital in Kerala
}

\author{
Selin Chiriyankandath Joy ${ }^{1}$, Anoosha Sunny², Meera Rajeshwari Nair ${ }^{3}$, Shikha Mariya Johnn, Shailaja Thumbarapully \\ Sukumaran ${ }^{5}$, Annamalai Pandurangan ${ }^{6}$, Prashant Chandra7 ${ }^{7}$, Vineetha Sajeesh ${ }^{8}$ \\ 1, 2, 3, 4, 8 Department of Pharmacy Practice, Nehru College of Pharmacy, Pampady, Thrissur, Kerala, India. ${ }^{5}$ Department \\ of Microbiology, P K DAS Institute of Medical Sciences, Vaniamkulam, Palakkad, Kerala, India. ${ }^{6}$ Department of \\ Pharmacy, Maharishi Markandeshwar (Deemed to Be University), Ambala, Haryana, India. ${ }^{7}$ Department of Pharmacy \\ Practice, Manipal College of Pharmaceutical Sciences, MAHE, Manipal, Udupi, Karnataka, India.
}

\section{ABSTRACT}

\section{BACKGROUND}

An antibiogram is an overall profile of the antibiotic susceptibility of an organism to a collection of antimicrobial agents routinely tested and used. Most hospitals issue once a year an antibiogram, which is a summary of the most important antibiotic resistance pattern of that hospital. This study aims to develop an antibiogram for a tertiary care hospital through a retrospective analysis. We also wanted to determine the antimicrobial resistance pattern of bacterial isolates from different clinical samples.

\section{METHODS}

This is a retrospective, hospital record-based study conducted at the Department of Microbiology in a tertiary care hospital from February 2018 to July 2018. A total of 890 samples (193 pus samples, 225 sputum samples 59 blood samples and 413 urine samples) was isolated and tested for antibiotic susceptibility by Kirby-Bauer discdiffusion method according to CLSI guidelines. WHONET software is used for the preparation of antibiogram report.

\section{RESULT}

The most frequently isolated species from pus samples were Gram positive cocci ( 42 $\%$ ) which shows highest sensitivity to Linezolid (93.5 \%). Among the urine samples, E. coli was the predominant isolate, which shows highest sensitivity towards Chloramphenicol (95.4\%) and Meropenem (92.8\%). In the 225 sputum samples, NFGNB (Non-Fermented Gram-Negative Bacteria) (37\%) was the most frequently isolated species which shows highest sensitivity towards Chloramphenicol. Out of the 59 blood samples, NFGNB shows highest sensitivity towards Linezolid (100 \%).

\section{CONCLUSION}

This hospital-based data will help to implement better infection-control strategies and improve the knowledge of antibiotic resistance pattern in our region.

\section{KEY WORDS}

Antimicrobial Resistance, Antibiogram, Non-Fermented Gram-Negative Bacteria

\author{
Corresponding Author: \\ Vineetha Sajeesh, \\ Department of Pharmacy Practice, \\ Nehru College of Pharmacy, \\ Kerala, Pampady, Thrissur, \\ Kerala, India. \\ E-mail: vineetha500@gmail.com
}

DOI: $10.14260 / \mathrm{jemds} / 2020 / 831$

How to Cite This Article:

Joy SC, Sunny A, Nair MR, et al. Antibiogram and antimicrobial susceptibility pattern of bacterial isolates from a tertiary care hospital in Kerala. J Evolution Med Dent Sci 2020;9(50):3787-3793, DOI: $10.14260 / \mathrm{jemds} / 2020 / 831$

Submission 29-07-2020,

Peer Review 03-10-2020,

Acceptance 09-10-2020,

Published 14-12-2020.

Copyright (C) 2020 Selin Chiriyankandath Joy et al. This is an open access article distributed under Creative Commons Attribution License [Attribution 4.0 International (CC BY 4.0)] 


\section{BACKGROUND}

Antimicrobial resistance (AMR) has become a major public health concern and is a phenomenon in which the originally sensitive antibiotics become ineffective against the microorganisms. ${ }^{1}$ The infection caused by the resistant strains of bacteria often increase the mortality, morbidity and cost of treatment than those caused by the susceptible strains. The emergence of AMR in addition to deficiency of novel antibiotics underlines the urgency of optimising the use of available antibiotics to reduce the disease burden. ${ }^{2}$ AST (Antimicrobial Susceptibility Testing) helps to determine AMR pattern of various bacterial isolates, to make the antibiotic treatment decisions and forecast therapeutic outcome. AST results are interpreted using the CLSI (Clinical and Laboratory Standards Institute) guidelines and it helps the clinicians to choose a cost-effective antibiotic to the patient. As the AMR pattern exposed an increase in the frequency of antibiotic resistant bacteria in various health-care settings, the perception of antibiograms was introduced. Antibiograms are useful in choosing the best effective therapy for the patients in need. It guides the health care professionals in finalising an appropriate empirical therapy in accordance with the local susceptibility rates. The susceptibility and resistance rates of the commonly isolated organisms to their commonly prescribed antibiotics are obtainable separately in the antibiogram. Susceptibility statistics varies geographically, and these susceptibility patterns of the clinically important isolates are vital for optimising antibiotic therapy and also helps to develop an effective infection control programme. ${ }^{3}$ The antibiogram report can be generated using a software called WHONET, which gives uniform guidelines in performing the AST. The potential of antibiograms would be the merging of patient related data to make the results more reliable. Antibiograms also aid in the stratification of the susceptibility percentage data for multi-drug resistant organisms and hence, ensures the quality of treatment provided. The most vital objective of antibiogram is the execution of antimicrobial stewardship by developing an antibiotic policy and standard treatment guidelines. AMR can also be monitored using this programme.

In the current study, we aim to determine the AMR pattern of bacterial isolates from a tertiary care hospital through a retrospective analysis, which helps to formulate an institutional antibiotic policy and thereby guides clinicians to choose an empirical antimicrobial therapy in a rational way.

\section{METHODS}

A retrospective study was carried out from February 2018 to July 2018, in the Department of Microbiology at a tertiary care hospital in Kerala. All the culture positive samples received in the Bacteriology section of the hospital was included in the study and the repeat isolates from a same person is excluded from the study in order to avoid duplication. The clinical samples like sputum, urine, pus and blood samples were collected from the patients and sent to the microbiology department for culture and sensitivity test. A retrospective evaluation of all the samples was carried out on the basis of the patient's case records, in a standard data collection format.

\section{Sample Processing}

The samples are inoculated on to blood agar and MacConkey agar. Plates are incubated at $37^{\circ} \mathrm{C}$ for 24 hours. Following incubation, isolated colonies obtained are identified by series of biochemical tests following standard procedures. All isolates were tested for AST by the standard Kirby-Bauer disc diffusion method according to Bauer et al. The test organism was picked up with a sterile loop, suspended in peptone water, and incubated at $37^{\circ} \mathrm{C}$ for 2 hours. It was then inoculated on the surface of a Muller-Hinton Agar (MHA) plate using sterile cotton swab. Antibiotics disc used in the study are Ampicillin (AMP), Amikacin (AMK), Erythromycin (ERY), AmoxicillinClavulanic acid (AMC), Vancomycin (VAN), Penicillin (PEN), Piperacillin- Tazobactam (TZP), Piperacillin (PIP), Ofloxacin (OFX), Norfloxacin (NOR), Nitrofurantoin (NIT), Meropenem (MEM), Linezolid (LNZ), Levofloxacin (LVX), Imipenem (IPM), Gentamycin (GEN), Clindamycin (CLI), Ciprofloxacin (CIP), Ampicillin-Sulbactam (SAM), Cefotaxime (CTX), Cefuroxime (CXM), Ceftazidime (CAZ), Tigecycline (TGC), Tetracycline (TCY), Teicoplanin (TEC), Trimethoprim-Sulfamethoxazole (SXT), Colistin (COL), Chloramphenicol (CHL), Cefpodoxime (CPD), Ceftriaxone (CRO), Cephalothin (CEP), Cefazolin (CZO). The plate was incubated at $37^{\circ} \mathrm{C}$ overnight. The zone of inhibition was measured and interpreted according to the CLSI guidelines. ${ }^{4}$

\section{Statistical Analysis}

Data was entered and organised into Microsoft Excel 2013 datasheet, then subjected to statistical analysis using the software WHONET (version 5.6). WHONET is a windowsbased database software package for the management of microbiology laboratory data and the analysis of AST results. ${ }^{5}$ The data structure has 3 main parts;

1. A laboratory configuration file: Which permits the entry and modification of laboratory specific information.

2. An interface for data entry: Which supports the entry of susceptibility results performed by disc diffusion, MIC etc. The results were interpreted as i.e., Resistant (R), Intermediated (I), Sensitive (S).

3. Analysis and reporting of resistance data: The user can select the type of analysis to run, the species of bacteria to analyse the subsets of isolates to include, the antimicrobial agents, period to examine type of analysis including the percentage of data categorised as Resistant, Intermediate, or Susceptible by standard break points. ${ }^{5}$

This study was approved by the hospital Ethics Committee (IEC No: IEC/NOV/18/05).

\section{RESULTS}

Out of 890 samples collected, 438 were males and 452 were females, 793 were from hospitalised inpatients and 97 samples from outpatient department. Other Enterobacteriaceae includes Enterobacter, E. coli, Salmonella, Klebsiella pneumonia, Proteus mirabilis, Proteus vulgaris, Citrobacter species, Citrobacter koseri. Non-Fermenting Gram Negative Bacilli (NFGNB) consists of Acinetobacter, Moraxella, Pseudomonas species, Pseudomonas aeruginosa, Stenotrophomonas, Burkholderia, Moraxella catarrhalis. GramPositive Cocci (GPC) include Staphylococcus aureus, 
Streptococcus pneumonia, Streptococcus pyogenes, Enterococcus species.

\section{Sensitivity Pattern of Different Pathogens to Different Antibiotics Based on Samples Collected.}

\section{Pus}

Among 890 samples collected, 193 were found to be pus samples. Out of which the most frequently isolated species were GPC 81 (42\%) followed by other Enterobacteriaceae 43 (22\%), Escherichia coli (E. coli) 37 (19\%) and NFGNB 32 (17 \%) (Fig. 1).

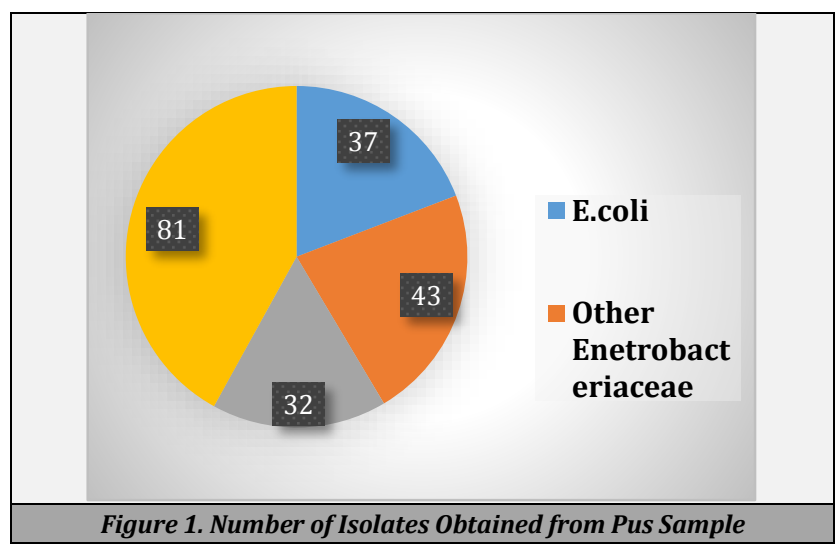

\begin{tabular}{|c|c|c|c|c|}
\hline $\begin{array}{l}\text { Antibiotics } \\
\text { Used }\end{array}$ & E. coli & $\begin{array}{c}\text { Other } \\
\text { Enterobacteriaceae }\end{array}$ & NFGNB & GPC \\
\hline CPD & - & - & - & 50 \\
\hline AMP & 5.05 & 31.4 & 50 & 80 \\
\hline SAM & 51.2 & 62.6 & 55.5 & 39.5 \\
\hline AMK & 63.1 & 66.1 & 74.7 & 33 \\
\hline AMC & 17.3 & 40.3 & 60 & 61.1 \\
\hline CEP & - & 0 & - & - \\
\hline CXM & 18.2 & 53.1 & 65 & 14.9 \\
\hline CRO & 15.5 & 45.5 & 65 & 14.9 \\
\hline CPX & 16.5 & 53.1 & 65 & 43.3 \\
\hline $\mathrm{COL}$ & - & - & - & - \\
\hline CIP & 27.6 & 65.1 & 74.7 & 25 \\
\hline CLI & - & - & - & - \\
\hline CZO & 13.2 & 21.5 & 20 & 13.7 \\
\hline CAZ & 18 & 56.1 & 75.5 & 14.1 \\
\hline CHL & 95.4 & 85.7 & 94.4 & 25.6 \\
\hline SXT & 42.5 & 53.5 & 90 & 38.3 \\
\hline ERY & 16.6 & - & - & 12.5 \\
\hline GEN & 60.1 & 72.7 & 80.8 & 35.8 \\
\hline IPM & 53.9 & 65 & 88.8 & 29.1 \\
\hline LVX & 27.3 & 62.4 & 74.7 & 24.1 \\
\hline LNZ & 50 & 33.3 & 100 & 92.2 \\
\hline MEM & 92.8 & 72.4 & 100 & 39.5 \\
\hline NIT & 82.5 & 32.3 & 51.6 & 93 \\
\hline NOR & 27.6 & 55.1 & 44.7 & 15.8 \\
\hline OFX & 28.8 & 62.2 & 74.7 & 24.6 \\
\hline PIP & 13.5 & 54.4 & 79.4 & 36.8 \\
\hline TZP & 67.3 & 72.8 & 88.9 & 41.3 \\
\hline PEN & 3.1 & 21.3 & 10 & 20.4 \\
\hline TCY & 27.7 & - & 20 & 58.6 \\
\hline TGC & - & - & - & 33.3. \\
\hline TEC & 33.3 & - & - & 54.7 \\
\hline VAN & 33.3 & 100 & 20 & 95.8 \\
\hline
\end{tabular}

Among the isolates, E. coli showed highest sensitivity towards MEM (100 \%), TGC (97.6\%) and CHL (96.6\%), moderate sensitivity towards IPM (56.15) and LVX (50.75\%) and least sensitivity towards AMP (6.5\%), CLI (5.5 \%) and PEN (2.3\%). Other Enterobacteriaceae showed high sensitivity towards CTX (97.9\%), TZP (96.5\%) and TGC (93.3\%), moderate sensitivity towards AMP (55\%), CHL (54.8\%) and least sensitivity towards PEN (24.2\%), NIT (16.6 \%). NFGNB showed high sensitivity towards CHL (79.1\%), TGC (75\%), moderate sensitivity towards SAM (41.6\%), PIP (54.8\%) and least sensitivity towards LNZ (16.6\%). GPC showed high sensitivity towards LNZ (93.5\%), moderate sensitivity towards IPM (50 \%) and least sensitivity towards CHL (25\%). (Table 1)

\section{Urine}

Among the 890 samples, 413 samples were found to be urine samples. Out of the total samples, predominant isolate was $E$. coli 222 (54\%) followed by other Enterobacteriaceae 137 (33 \%), NFGNB 35 (8\%) and GPC 19 (5\%).

\begin{tabular}{|c|c|c|c|c|}
\hline Antibiotics Used & E. coli & Other Enterobacteriaceae & NFGNB & GPC \\
\hline CPD & - & - & - & 50 \\
\hline AMP & 5.05 & 31.4 & 50 & 80 \\
\hline SAM & 51.2 & 62.6 & 55.5 & 39.5 \\
\hline AMK & 63.1 & 66.1 & 74.7 & 33 \\
\hline AMC & 17.3 & 40.3 & 60 & 61.1 \\
\hline CEP & - & 0 & - & - \\
\hline CXM & 18.2 & 53.1 & 65 & 14.9 \\
\hline CRO & 15.5 & 45.5 & 65 & 14.9 \\
\hline CTX & 16.5 & 53.1 & 65 & 43.3 \\
\hline $\mathrm{COL}$ & - & - & - & - \\
\hline CIP & 27.6 & 65.1 & 74.7 & 25 \\
\hline CLI & - & - & - & - \\
\hline $\mathrm{CZO}$ & 13.2 & 21.5 & 20 & 13.7 \\
\hline CAZ & 18 & 56.1 & 75.5 & 14.1 \\
\hline $\mathrm{CHL}$ & 95.4 & 85.7 & 94.4 & 25.6 \\
\hline SXT & 42.5 & 53.5 & 90 & 38.3 \\
\hline ERY & 16.6 & - & - & 12.5 \\
\hline GEN & 60.1 & 72.7 & 80.8 & 35.8 \\
\hline IPM & 53.9 & 65 & 88.8 & 29.1 \\
\hline LVX & 27.3 & 62.4 & 74.7 & 24.1 \\
\hline LNZ & 50 & 33.3 & 100 & 92.2 \\
\hline MEM & 92.8 & 72.4 & 100 & 39.5 \\
\hline NIT & 82.5 & 32.3 & 51.6 & 93 \\
\hline NOR & 27.6 & 55.1 & 44.7 & 15.8 \\
\hline OFX & 28.8 & 62.2 & 74.7 & 24.6 \\
\hline PIP & 13.5 & 54.4 & 79.4 & 36.8 \\
\hline TZP & 67.3 & 72.8 & 88.9 & 41.3 \\
\hline PEN & 3.1 & 21.3 & 10 & 20.4 \\
\hline TCY & 27.7 & - & 20 & 58.6 \\
\hline TGC & - & - & - & 33.3 \\
\hline TEC & 33.3 & - & - & 54.7 \\
\hline VAN & 33.3 & 100 & 20 & 95.8 \\
\hline
\end{tabular}

Among the isolates, E. coli showed high sensitivity towards CHL (95.4\%), MEM (92.8\%), moderate sensitivity towards IPM (53.9\%), SXT (42.5\%) and least sensitivity towards AMP (5.05\%), PEN (3.1\%). Other Enterobacteriaceae showed high sensitivity towards VAN (100\%), CHL (85.7\%), moderate sensitivity towards AMC (40.3\%), CRO (45.5\%) and least sensitivity towards PEN (21.3\%), CZO (21.5\%). NFGNB showed high sensitivity towards LNZ (100\%), MEM (100\%) and CHL (94.4\%), moderate sensitivity towards NOR (44.7\%), AMP (50 \%) and least sensitivity towards PEN (10 \%). GPC showed high sensitivity towards VAN (95.8\%), NIT (93\%) and LNZ (92.2\%), moderate sensitivity towards CTX (43.3\%), TZP (41.3\%), and least sensitivity towards ERY (12.5\%), CZO (13.7\%) (Table 2).

\section{Sputum}

Among 890 samples, 225 were found to be sputum samples in which NFGNB 84 (37\%) was the most frequently isolated followed by other Enterobacteriaceae 79 (35\%), GPC 35 (16 $\%)$ and E. coli 27 (12\%). Among the isolates, E. coli showed high sensitivity towards CHL (95.8\%), moderate sensitivity towards IPM (46.5\%), TCY (45.8\%), and least sensitivity towards PEN (6.2 \%), PIP (2.9 \%). Other Enterobacteriaceae showed high sensitivity towards COL (100\%), MEM (97.5), moderate sensitivity towards TEC (58.3\%) and least sensitivity towards CLT (16.6\%) AMP (10.4\%). NFGNB 
showed high sensitivity towards GEN (95.3\%), CIP (90.2\%), moderate sensitivity towards TGC (51\%), CXM (53.8\%) and least sensitivity towards AMP (27.2\%), PEN (27.2\%). GPC showed high sensitivity towards VAN (90\%), moderate sensitivity towards NIT (40.8\%), CAZ (42.7\%) and least sensitivity towards OFX (20\%). (Table 3).

\begin{tabular}{|c|c|c|c|c|}
\hline Antibiotics Used & E. coli & $\begin{array}{c}\text { Other } \\
\text { Enterobacteriaceae }\end{array}$ & NFGNB & GPC \\
\hline CPD & - & - & - & - \\
\hline AMP & - & 10.4 & 27.2 & 64.4 \\
\hline SAM & 44.4 & 67.5 & 55.4 & 49.4 \\
\hline AMK & 66.6 & 92.5 & 72.5 & 55.6 \\
\hline AMC & 20.8 & 66.1 & 79.6 & 62.3 \\
\hline CEP & - & - & - & - \\
\hline CXM & 20.8 & 73.4 & 53.8 & 38.6 \\
\hline CRO & 20.8 & 79.5 & 65.6 & 73.7 \\
\hline CTX & 20.8 & 80.2 & 79.4 & 77.2 \\
\hline $\mathrm{COL}$ & - & 100 & - & - \\
\hline CIP & 31.9 & 82.3 & 90.2 & 53.5 \\
\hline CLI & - & 16.6 & - & 64.2 \\
\hline CZO & 20.8 & 70.6 & 27.7 & 47.9 \\
\hline CAZ & 11.1 & 70.2 & 62.1 & 42.7 \\
\hline CHL & 95.8 & 77.4 & 87.2 & 29.3 \\
\hline SXT & 43.7 & 82.4 & 76.5 & 68.7 \\
\hline ERY & - & - & - & 38.9 \\
\hline GEN & 54.1 & 91 & 95.3 & 56.1 \\
\hline IPM & 46.5 & 91.8 & 66.1 & 55.4 \\
\hline LVX & 31.9 & 85.8 & 87.8 & 53.5 \\
\hline LNZ & - & - & - & 95 \\
\hline MEM & 80.5 & 97.5 & 83.6 & 45.6 \\
\hline NIT & - & - & - & 40.8 \\
\hline NOR & - & - & - & - \\
\hline OFX & - & - & - & 20 \\
\hline PIP & 2.7 & 75.8 & 84.9 & 29.5 \\
\hline TZP & 75.7 & 97.1 & 83.9 & 46.9 \\
\hline PEN & 6.2 & 76 & 27.2 & 61.7 \\
\hline TCY & 45.8 & 80.8 & 59.1 & 74.9 \\
\hline TGC & 83.3 & 95.8 & 51 & 63.3 \\
\hline TEC & 16.6 & 58.3 & - & 61.1 \\
\hline VAN & - & - & - & 90 \\
\hline
\end{tabular}

\section{Blood}

Among 890 samples, 59 were found to be blood samples, out of which NFGNB 36 (61\%) were the most frequently isolated species followed by Other Enterobacteriaceae 34 (57.6\%), GPC 19 (32\%) and E. coli 12 (20.3\%).

\begin{tabular}{|c|c|c|c|c|}
\hline Antibiotics Used & E. coli & $\begin{array}{c}\text { Other } \\
\text { Enterobacteriaceae }\end{array}$ & NFGNB & GPC \\
\hline CPD & - & - & - & 41.6 \\
\hline AMP & - & 37.5 & 27.3 & 54.1 \\
\hline SAM & 50 & 56.2 & 50.1 & 63.8 \\
\hline AMK & 80 & 82.9 & 90.7 & 87.5 \\
\hline AMC & - & 44.5 & 31.7 & 80.5 \\
\hline CEP & - & - & - & - \\
\hline CXM & - & 38.3 & 16.2 & 70.8 \\
\hline CRO & - & 42.4 & 32.9 & 80.5 \\
\hline CTX & - & 42.4 & 32.9 & 80.5 \\
\hline COL & - & - & - & - \\
\hline CIP & - & 74.4 & 86.2 & 84.3 \\
\hline CLI & 30 & - & - & 75 \\
\hline CZO & - & 37.5 & 13.3 & 100 \\
\hline CAZ & - & 62 & 50.5 & 70.8 \\
\hline CHL & - & 54.5 & 72.2 & - \\
\hline SXT & 80 & 55.5 & 42.2 & 100 \\
\hline ERY & - & - & - & 75 \\
\hline GEN & - & 61.6 & 92.5 & 84.3 \\
\hline IPM & 90 & 77 & 91.6 & 62.5 \\
\hline LVX & 50 & 74.4 & 77.9 & 83.3 \\
\hline LNZ & 30 & 33.3 & 100 & 93.7 \\
\hline MEM & - & 63.3 & 93.3 & 62.5 \\
\hline NIT & 80 & 37.5 & 13.3 & 100 \\
\hline NOR & - & 37.5 & 13.3 & - \\
\hline OFX & 10 & 37.5 & 13.3 & - \\
\hline PIP & - & 40.8 & 75.7 & 58.3 \\
\hline TZP & - & 79.9 & 80 & 70.8 \\
\hline PEN & 70 & 66.6 & 0 & 40.6 \\
\hline TCY & - & - & - & 75 \\
\hline TGC & - & - & - & 100 \\
\hline TEC & - & - & - & 83.3 \\
\hline VAN & - & - & - & 100 \\
\hline
\end{tabular}

Among the isolates, E. coli showed high sensitivity towards IPM (90\%), moderate sensitivity towards SAM (50\%) and least sensitivity towards OFX (10 \%). Other Enterobacteriaceae showed high sensitivity towards TZP (79.9 \%), moderate sensitivity towards CHL (54.5\%), SXT (55.5\%) and least sensitivity towards LNZ (33.3\%). NFGNB showed high sensitivity towards LNZ (100\%), MEM (93.3 \%), moderate sensitivity towards SAM (50.1\%), CAZ (50.5\%) and least sensitivity towards NIT (13.3\%), NOR (13.3\%) and OFX (13.3\%). GPC showed high sensitivity towards CZO (100\%), SXT (100\%), NIT (100\%) and VAN (100\%), moderate sensitivity towards AMP (54.1\%), least sensitivity towards PEN (40.6 \%), CPD (41.6\%) (Table 4).

\section{DISCUSSION}

In our study, 890 samples were collected from various departments, out of which, 438 were male patients and 452 were female patients, contradicting the study findings of Saritha Kammath et al., where male patients (62.3\%) where found to be higher than female patients $(37.7 \%) .{ }^{6}$ Among the 413 urine samples collected, E.coli (54 \%) was the predominant organism isolated followed by other Enterobacteriaceae (33\%), NFGNB (8\%) and GPC (5\%).These findings were consistent with the study done by Nasim Kashef et al. ${ }^{7}$ In our study, E. coli showed high susceptibility towards Chloramphenicol (95.4\%) similar to the study conducted by Beyene et al. ${ }^{8}$ The sensitivity pattern showed moderate sensitivity towards trimethoprim-sulphamethoxazole $(42.5$ $\%$ ) in accordance with the study conducted by Nasim Kashef et al. ${ }^{21}$

E. coli was least sensitive to ampicillin, similar finding was reported by Getenet Beyene et al. ${ }^{8}$ Enterobacteriaceae were the next common group of organisms isolated. A study conducted by Shanthi $J$ et al showed high susceptibility of Enterobacteriaceae to vancomycin as that obtained from our study. ${ }^{9}$ The study findings of Wahengbam PS Al Waheed et al showed moderate susceptibility towards netilmicin (68\%) contradicting our study in which ampicillin (55\%) was moderately susceptible.10

Maximum resistance to third generation cephalosporins was reported by A. Ashaet al., which is in accordance with our study findings ${ }^{11}$ Contrary to our study, NFGNB species were highly susceptible to meropenem (100\%), a study by Shilpa A. Gokale produced similar results.12 NFGNB species showed moderate susceptibility towards piperacillin (54.8\%) similar to the study findings of Wahengbam PS Al Waheed et al. ${ }^{10}$ The isolates showed least susceptibility to ampicillin (2.3\%) and cotrimoxazole $(9.2 \%)$ in a study conducted by Shilpa A. Gokaleet. al., contrary to our study in which penicillin (10\%) showed least susceptibility and ampicillin (50 \%) was moderately sensitive. ${ }^{12}$ In accordance with our study, Gram positive cocci were highly sensitive towards vancomycin in the findings of Ahish Jitendranath et al.13

Ochedaet. al., in their study showed high susceptibility of gram-positive cocci towards erythromycin (66.7 \%) contradicting our results in which erythromycin showed least susceptibility (12.5\%). ${ }^{14}$ Gram positive cocci was found to have moderate sensitivity towards cefotaxime $(43.3 \%)$ and piperacillin with tazobactam $(41.3 \%)$ in contrast to the results 
obtained by Ocheda et al.14 Among the 225 sputum samples collected, NFGNB (37 \%) was the most frequent isolate followed by other Enterobacteriaceae (35\%), GPC (12\%) and E.coli (12\%) unlike the study conducted by Syed Muteq Ahmed et al in which Enterobacteriaceae (41.9) was predominant followed by NFGNB $(26.8 \%) .{ }^{15}$ E. coli showed high sensitivity to imipenem (100\%) as observed by Gurumayum Preethiet al., contradicting our findings in which the organism showed moderate sensitivity (46.5\%) towards the antibiotic. ${ }^{16}$

Least susceptibility was shown towards piperacillin (2.9 $\%$ ) by the organism as observed by Ugur Gonlugur et. al., where the percentage sensitivity was 27.8 .17 In the study findings of Ravi Pathiyil Shake et. al., least susceptibility was observed towards chloramphenicol by $E$. coli, this result contradicts our study where maximum susceptibility towards chloramphenicol (95.8\%) was seen. Enterobacteriaceae isolates showed high susceptibility towards meropenem (97.5 $\%)$ consistent with the findings of Gurumayum Preethi et. al. ${ }^{16}$ According to the study results of Namratha KG et al., Enterobacteriaceae showed least susceptibility towards ampicillin (10.4\%) as seen in our study. ${ }^{18}$

Enterobacteriaceae showed moderate sensitivity towards teicoplanin (58.3\%) whereas the study findings of Namratha KG et. al., and Eze E.et al., showed moderate sensitivity of the organisms towards ciprofloxacin (59\%). ${ }^{19}$ The study findings of Maria Amaushtaq Gill et al., and Gurumayum Preethi et al., where NFGNB isolates showed least (12.2\%) and moderate (48.8\%) susceptibility to ciprofloxacin contradicts our study in which ciprofloxacin was found to have high susceptibility (90.2\%).16 Moderate susceptibility was shown by NFGNB towards ciprofloxacin (50.4\%) and amikacin (49.5\%) in the study findings of Shilpa K. Gokaleet. al., contrary to our study where cefuroxime $(53.8 \%)$ and tigecycline $(51 \%)$ were found to have moderate susceptibility. ${ }^{12}$

NFGNB showed least susceptibility towards ampicillin $(27.2 \%)$, similar results were obtained from the study done by ShilpaK. Gokale et al.12 Gram positive isolates showed high sensitivity towards vancomycin $(90 \%)$ in accordance with the findings of Tripathi Purti C et al. ${ }^{20}$ In correlation with the study findings of C. Manikandan et al Gram positive isolates exhibited least sensitivity towards ofloxacin $(20 \%) .{ }^{21} \mathrm{~A}$ study conducted by Borkot Ullah et al., summarised Gram positive isolates to have least susceptibility towards ceftazidime whereas ceftazidime was found to be moderately susceptible ( $42.7 \%$ ) in our study. ${ }^{22}$

Out of 59 blood samples collected, NFGNB (61\%) was found to be the most frequent isolate followed by other Enterobacteriaceae (57.6\%), GPC (32\%) and E. coli (20.3\%) similar to the study findings of Narayan Gyawali et al., in which Staphylococcus aureus (39.6\%) remained the predominant isolate followed by Klebsiella $(13.5 \%)$. $^{23}$

E. coli isolates showed high susceptibility towards imipenem (90\%) similar to the study findings of Ali Hashemiet al. ${ }^{15}$ In the study conducted by Sweta et al. E. coli was found to be moderately susceptible towards ofloxacin (50 \%) contrary to our study where ofloxacin showed least (10 \%) susceptibility. ${ }^{24}$ The study findings of Shilpi Gupta et al. revealed moderate susceptibility of E.coli towards ciprofloxacin (52.8\%) contradicting our study findings where ampicillin / sulbactum (50\%) was moderately susceptible. ${ }^{25}$
Enterobacteriaceae isolates showed high susceptibility to piperacillin / tazobactam (79.9\%), the study findings of Shilpi Gupta et al. contradicts our findings where high susceptibility was shown towards imipenem. A study conducted by Tariq Mahmud Tariq revealed moderate susceptibility of Enterobacteriaceae towards gentamycin (47.2\%), contrary to our results where chloramphenicol was found to be moderately (54.5\% susceptible. ${ }^{26}$

Shilpi Gupta et al. in this study showed least susceptibility of Enterobacteriaceae isolates towards ampicillin unlike our study in which linezolid (33.3\%) was found to be least susceptible. NFGNB isolates showed high susceptibility towards linezolid $(100 \%)$ contrary to the study findings of Shilpi Gupta et al. where colistin showed high (100\%) susceptibility.

Moderate sensitivity to co-trimoxazole ( $48 \%$ ) was shown by the study conducted by Narayan Gyawaliet al., unlike our study where ampicillin / sulbactum was found to be moderately ( $50.1 \%$ ) susceptible. NFGNB isolates were found to have least susceptibility towards nitrofurantoin (13.3\%) which is in accordance with the study findings of Shankara Kutty J et al. Gram-positive isolates showed high susceptibility towards vancomycin (100\%) similar to the study findings of Tariq Mahmud Tariq.

Moderate susceptibility was shown towards cefotaxime by Gram positive cocci in a study conducted by Naryan Gyawali et al. unlike our study results where ampicillin (54.1\%) was found to be moderately susceptible. Gram positive isolates showed least susceptibility towards penicillin similar to the study findings of Narayan Gyawali et al. ${ }^{27}$

Out of the 193 pus samples collected, GPC (42\%) was the predominant isolate followed by Enterobacteriaceae (22 \%), E.coli (19 \%) and NFGNB (17\%) contradicting the study findings of Rugira Trojan et al. in which E.coli (51.2 \%) was the most frequent isolate. ${ }^{28} \mathrm{In}$ the study conducted by Satyjee $\mathrm{K}$ Pawaret al., high sensitivity was shown towards meropenem similar to our study. ${ }^{29}$ Imipenem (56.15\%) was found to be moderately sensitive towards E.coli contrary to the study findings of Roopa C et al., where imipenem showed high (94.4 $\%)$ sensitivity. ${ }^{30}$ E. coli showed least susceptibility towards ampicillin (6.5\%) similar to the study findings of Roopa C et al. ${ }^{30}$ Enterobacteriaceae isolates showed high susceptibility towards piperacillin / tazobactam (96.5\%) in accordance with the study findings of Rajiv Kumar et al.

Moderate sensitivity was shown towards piperacillin (54.8 \%) similar to the results obtained by Swati Duggal et al., where piperacillin showed percentage sensitivity of $61.29 .{ }^{31}$ In the study conducted by Satyajeet et al. Enterobacteriaceae isolates showed least susceptibility to nitrofurantoin (25\%) which is in accordance with our study. NFGNB isolates showed high susceptibility to chloramphenicol $(79.1 \%)$ contrary to the results obtained by Swati Duggal et al. in which imipenem was found to be highly susceptible. Moderate susceptibility was shown by NFGNB isolates towards piperacillin (54.8\%) as in a study conducted by A. Malini et al. ${ }^{32}$ NFGNB isolates showed least susceptibility towards Linezolid contradicting the study findings of Swathi Duggalet al., where cefadroxil (2.1\%) was least susceptible.

Gram positive isolates showed high susceptibility towards linezolid (93.5\%) in accordance with the study results obtained by Poonamvarma et al. ${ }^{33}$ A study conducted by Swati Duggal et al. showed moderate sensitivity of Gram-positive 
isolates towards imipenem, similar to our study results. Gram positive isolates showed least susceptibility towards chloramphenicol (25\%) in accordance with the results obtained by Narayan Gyawali et al.

\section{CONCLUSIONS}

The study identifies the sensitivity pattern of organisms towards the commonly used antibiotics. The cause of resistance maybe attributed to factors like irrational use of antibiotics. Hence to prevent the resistant bacteria, it is essential to have an antibiogram for hospitals to know the susceptibility pattern of common organisms and to provide suitable empirical therapy.

Data sharing statement provided by the authors is available with the full text of this article at jemds.com.

Financial or other competing interests: None.

Disclosure forms provided by the authors are available with the full text of this article at jemds.com.

We express our sincere gratitude to the staff of Department of Pharmacy Practice, Nehru College of Pharmacy, for their constant support. We also thank the Department of Microbiology, PK DAS Institute of Medical Sciences for their immense support in completing our project.

\section{REFERENCES}

[1] Amin ET, Njumkeng C, Kika BT, et al. Pattern of antimicrobial resistance among bacterial isolates from urogenital clinical specimens: a descriptive study from the Buea Health District, Cameroon. Drugs Real World Outcomes 2018;5(2):101-8.

[2] Zur Wiesch PA, Kouyos R, Abel S, et al. Cycling empirical antibiotic therapy in hospitals: meta-analysis and models. PLoS Pathog 2014;10(6):e1004225.

[3] Joseph B, Sheeba SN, Sujatha S, et al. Study of antibiogram and drug resisyance for some bacterial strains from the Human internal fluid (CSF, Ascitic Fluid and Synovial Fluid). International Journal of Pharmacology 2011;7(4):463-70.

[4] Binkley S, Fishman NO, LaRosa LA, et al. Comparison of unit-specific and hospital-wide antibiograms: potential implications for selection of empirical antimicrobial therapy. Infect Control Hosp Epidemiol 2006;27(7):6827.

[5] Joshi S. Hospital antibiogram: a necessity. Indian J Med Microbiol 2010;28(4):277-80.

[6] Kamath S, Mallayya S, Shenoy S, et al. Nosocomial infections in neonatal intensive care units: profile, risk factor assessment and antibiogram. Indian J Pediatr 2010;77(1):37-9.

[7] Kashef N, Djavid GE, Shahbazi S. Antimicrobial susceptibility patterns of community-acquired uropathogens in Tehran, Iran. J Infect Dev Ctries 2010;4(4):202-6.

[8] Beyene G, Tsegaye W. Bacterial uropathogens in urinary tract infection and antibiotic susceptibility pattern in Jimma university specialized hospital, southwest Ethiopia. Ethiopian J Health Sci 2011;21(2):141-6.
[9] Shanthi J, Kayathri S. Incidence, distribution and antibiogram of uropathogens isolated from patients with urinary tract infections. Adv Appl Sci Res 2012;3(6):3410-4.

[10] Al-Waheed WPS, Kumar R, Wolfgang Viechtbauer, Sebastian Bonhoefferi. Antibiotics - is there a need for antibiogram? Majmaah Journal of Health Science 2014;2(1):47-53.

[11] Asha A, Karnaker VK, Rai R. Characterization and antibiogram of klebsiellae isolated from clinical samples. Int J Curr Microbiol Appl Sci 2017;6(7):386-96.

[12] Gokhale SK, Metgud SC. Characterization and antibiotic sensitivity pattern of non-fermenting gram negative bacilli from various clinical samples in a tertiary care hospital, Belgaum. J Pharmaceutical \& Biomedical Sciences 2012;17:1-5.

[13] Jithendra A, Radhika R, Bhargavi L, et al. Microbiological profile of urinary tract infection in pediatric population from a tertiary care hospital in South Kerala. J Bacteriol Mycol Open Access 2015;1(1):4-7.

[14] Ochada NS, Nasiru IA, Thairu Y, et al. Antimicrobial susceptibility pattern of urinary pathogens isolated from two tertiary hospitals in southwestern Nigeria. African Journal of Clinical and Experimental Microbiology 2014;16(1):12-22.

[15] Shams S, Hashmi A, Esmkhani M, et al. Imipenem resistance in clinical Escherichia Coli from Qom, Iran. BMC Resnotes 2018;11:314.

[16] Preeti G, Laifangbam S. Aerobic bacteriological profile of AFB negative sputum samples at a tertiary care medical institute in Eastern India. Journal of Evolution of Medical and Dental Sciences 2016;5(58):4022-6.

[17] Gonlugur U, Backici MZ, Akkurt I, et al. Antibiotic susceptibility patterns among respiratory isolates of Gram-negative bacilli in a Turkish university hospital. BMC Microbiol 2004;4:32.

[18] Bengalorkar G, Kumar TN. Culture and sensitivity pattern of micro-organism isolated from diabetic foot infections in a tertiary care hospital. Int J Curr Biomed Phar Res 2011;1(2):34-40.

[19] Namratha KG, Shreeshma P, Subbannaya K, et al. Characterization and antibiogram of klebsiella spp isolated from clinical specimens in a rural teaching hospital. Scholars Journal of Applied Medical Sciences 2015;3(2E):878-83.

[20] Purti CT, Kiran D. Lower respiratory tract infections current etiological trendsand antibiograms. J Pharm Biomed Sci 2014;4(3):249-55.

[21] Manikandan C, Amsath A. Antibiotic susceptibility of bacterial strains isolated from patients with respiratory infections. Int J Pure Appl Zool 2013;1(1):61-9.

[22] Ullah B, Ahmed S, Shahariar M, et al. Current trend of antibiotic resistance in lower respiratory tract infections (LRTIs): an experience in a teaching hospital in Bangladesh. Bangladesh Pharmaceutical Journal 2016;19(1):85-91.

[23] Gyawalli N, Sanjana RK. Bacteriological profile and antibiogram of neonatal septicaemia. Indian J Paediatr 2012;80(5):371-4.

[24] Oza SS, Mehta S, Kikani K, et al. Bacteriological profile and antibiogram of blood culture isolates from patients of rural tertiary care hospital. Int J Micro Mycology 2016;4(3):1-7. 
[25] Guptha S, Kashyap B. Bacteriological profile and antibiogram of blood culture isolates from a tertiary care hospital of North India. Tropical Journal of Medical Research 2016;19(2):94.

[26] Tariq TM. Bacteriologic profile and antibiogram of blood culture isolates from a children's hospital in Kabul. J Coll Physicians Surg Pak 2014;24(6):396-9.

[27] Gyawali N, Sanjana RK. Bacteriological profile and antibiogram of neonatal septicemia. Indian J Pediatr 2012;80(5):371-4.

[28] Trojen R, Razdan L, Singh N. Antibiotic susceptibility patterns of bacterial isolates from pus samples in a tertiary care hospital of Punjab, India. Int J Microbiol 2016;2016:9302692.

[29] Pawar SK, Patil SR, Karande JS, et al. Antimicrobial susceptibility pattern of clinical isolates in intensive care unit in a tertiary care hospital from Western India. International Journal of Scientific Study 2016;4(2):10813.
[30] Roopa C, Deepali V. PUS culture isolates and their antibiotic sensitivity at a tertiary care hospital in Hyderabad Karnataka Region. International Journal of Medical Microbiology and Tropical Diseases 2017;3(4):140-5.

[31] Duggal S, Khatri PK, Parihar RS, et al. Antibiogram of various bacterial isolates from pus samples in a tertiary care centre in Rajasthan. International Journal of Science and Research 2015;4(5):1580-4.

[32] Malini A, Deepa EK, Gokul BN, et al. Nonfermenting gramnegative bacilli infections in a tertiary care hospital in Kolar, Karnataka. J Lab Physicians 2009;1(2):62-6.

[33] Verma P. Antibiotic sensitivity treatment for gram positive bacteria isolated from pus sample. Bulletin of Environment, Pharmacology and Life Science 2012;1(10):3-6. 